\title{
A space weather mission concept: observatories of the solar corona and active regions (oscar) - Erratum
}

Antoine Strugarek $^{1,2, *}$, Nils Janitzek ${ }^{3}$, Arrow Lee ${ }^{4}$, Philipp Löschl ${ }^{5}$, Bernhard Seifert ${ }^{6}$, Sanni Hoilijoki ${ }^{7,8}$, Emil Kraaikamp ${ }^{9}$, Alankrita Isha Mrigakshi ${ }^{3,10}$, Thomas Philippe ${ }^{11}$, Sheila Spina ${ }^{12}$, Malte Bröse ${ }^{13}$, Sonny Massahi ${ }^{14}$, Liam O’Halloran ${ }^{15}$, Victor Pereira Blanco ${ }^{16}$, Christoffer Stausland ${ }^{17}$, Philippe Escoubet $^{18}$, and Günter Kargl ${ }^{19}$

1 Département de physique, Université de Montréal, C.P. 6128 Succ. Centre-Ville, Montréal, QC H3C-3J7, Canada

*Corresponding author: strugarek@astro.umontreal.ca

2 Laboratoire AIM Paris-Saclay, CEA/Irfu Université Paris-Diderot CNRS/INSU, 91191 Gif-sur-Yvette, France

3 Institute of Experimental and Applied Physics, University of Kiel, Kiel, Germany

4 Mullard Space Science Laboratory, University College London, Dorking, United Kingdom

5 Institut für Astrophysik, University of Vienna, Vienna, Austria

${ }^{6}$ FOTEC - Department of Aerospace Engineering, Wiener Neustadt, Austria

Finnish Meteorological Institute, Helsinki, Finland

8 Department of Physics, University of Helsinki, Helsinki, Finland

9 Royal Observatory of Belgium, Brussels, Belgium

10 German Aerospace Centre (DLR), Institute of Aerospace Medicine, Cologne, Germany

11 Institut Supérieur de l'Aéronautique et de l'Espace, Toulouse, France

12 La Sapienza - University of Rome, Italy

13 Department of Physics, Free University of Berlin, Berlin, Germany

14 Danish Space Research Institute, Copenhagen, Denmark

15 School of Medicine and Medical Sciences, University College Dublin, Dublin, Ireland

16 Dpto. de Astrofísica y CC. de la Atmósfera, Universidad Complutense de Madrid, 28040 Madrid, Spain

17 Department of Physics, University of Oslo, Norway

18 ESA/ESTEC, Noordwijk, The Netherlands

19 Space Research Institute, Austrian Academy of Sciences, Austria

J. Space Weather Space Clim., 5 (2015) A4, DOI: 10.1051/swsc/2015003

\begin{abstract}
In this erratum we acknowledge EASCO as one of the inspirational mission concepts that helped the development of our original mission concept OSCAR.
\end{abstract}

It was brought to our attention that our original paper failed to acknowledge the mission concept EASCO, which was originally laid out in Gopalswamy et al. (2011). At the time we developed OSCAR, EASCO was one of the inspirational mission concepts on which we built our new and original concept of twin satellites leading and trailing the Earth with a separation angle of $68^{\circ}$. The omission of EASCO in our original paper was unintentional, and we wanted to acknowledge this important previous work in the present erratum.

\section{References}

Gopalswamy, N., J.M. Davila, O.C. St Cyr, E.C. Sittler, F. Auchere, et al. Earth-Affecting Solar Causes Observatory (EASCO): a potential International Living with a Star Mission from Sun-Earth L5. J. Atmos. Solar-Terr. Phys., 73 (5), 658663, 2011, DOI: 10.1016/j.jastp.2011.01.013, http://adsabs. harvard.edu/cgi-bin/nph-data_query?bibcode=2011JASTP..73 . 658G\&link_type=EJOURNAL.

Cite this article as: Strugarek A, Janitzek N, Lee A, Löschl P, Seifert B, et al. A space weather mission concept: observatories of the solar corona and active regions (oscar) - Erratum. J. Space Weather Space Clim., 7, A1, 2017, DOI: 10.1051/swsc/2016040. 